\title{
Photosynthetic profiling of a Dunaliella salina mutant DS240G-1 with improved $\beta$-carotene productivity induced by heavy ions irradiation
}

\author{
Yimei $\mathrm{Xi}^{1,3}$, Ying Liu ${ }^{2}$, Zhanyou $\mathrm{Chi}^{3}$, Liang Yin ${ }^{1}$, Lijuan Wang ${ }^{1}$, Guanghong Luo ${ }^{1 *}$ \\ (1. Gansu Engineering Technology Research Center for Microalgae, Hexi University, Zhangye 734000, Gansu, China; \\ 2. State Environmental Protection Key Laboratory of Drinking Water Source Management and Technology, \\ Shenzhen Academy of Environmental Science, Shenzhen 518001, Guangdong, China; \\ 3. School of Bioengineering, Dalian University of Technology, Dalian 116024, Liaoning, China)
}

\begin{abstract}
Carbon-ion irradiation is a technique for trait improvement in the microalgae, but the underlying mechanisms that how it altered the biomass, and photosynthetic pigments accumulation were unclear. One mutant (DS240G-1) was obtained from Dunaliella salina by heavy ion irradiation mutagenesis. Compared to the wild type, the biomass accumulation and maximum growth rate of DS240G-1 were increased by $34 \%$ and $55 \%$ respectively, and its $\beta$-carotene content was $21 \%$ higher than the wild type. Subsequent analysis of the chlorophyll fluorescence parameters indicated that higher $\beta$-carotene productivity was likely owing to the improved maximum quantum efficiency $\left(F_{v} / F_{m}\right)$ and decreased thermal dissipation of photosynthesis in DS240G-1 than that of wild type during cultivation. In addition, the result of this study revealed that high content of ROS may induce $\beta$-carotene accumulation in mutant DS240G-1. Also, the total fatty acid (TFA) content in mutant DS240G-1 was $79 \%$ higher than that in wild type. Owing to its high $\beta$-carotene productivity and total fatty acid content, DS240G-1 could be considered as a promising candidate for microalgae $\beta$-carotene and biodiesel production. This work provided the first insight into the biological effects involved in carbon-ions irradiation on the photosynthetic activity of $D$. salina.
\end{abstract}

Keywords: microalgae, D. salina, carbon-ions irradiation, chlorophyll fluorescence, $\beta$-carotene DOI: $10.25165 /$ j.ijabe.20211402.5993

Citation: Xi Y M, Liu Y, Chi Z Y, Yin L, Wang L J, Luo G H. Photosynthetic profiling of a Dunaliella salina mutant DS240G-1 with improved $\beta$-carotene productivity induced by heavy ions irradiation. Int J Agric \& Biol Eng, 2021; 14(2): 211-217.

\section{Introduction}

Microalgae have been considered as one of the major promising sources for third-generation biofuel production, due to their advantages such as short growth period, high photosynthesis efficiency, and high lipid content as well as the advantage that the co-production of valuable by-products ${ }^{[1-3]}$. The major compounds in microalgae involve fatty acid ${ }^{[4]}$, protein, soluble polysaccharides, carotenoids, etc. $\beta$-carotene is one of the powerful antioxidants among carotenoids with many applications in nutraceuticals, food and feed industries because of its anti-aging, anti-inflammatory, sun proofing, and immune system boosting effects on organisms ${ }^{[5]}$. The unicellular green microalgae Dunaliella salina, with $\beta$-carotene content of $0.3 \%-10 \%$, is recognized as the best biological source of natural $\beta$-carotene, and currently this species is the only commercialized microalgae for $\beta$-carotene production ${ }^{[6]}$. From a commercial point of view, the best strains of $D$. salina in mass cultivation

Received date: 2020-07-01 Accepted date: 2021-02-24

Biographies: Yimei Xi, PhD, research interests: microalgae biotechnology, Email:970398787@mail.dlut.edu.cn; Ying Liu, PhD, research interests: microalgae biotechnology, Email: liuying@sz.pku.edu.cn; Zhanyou Chi, PhD, Professor, research interests: microalgae biotechnology, Email: chizhy@ dlut.edu.cn; Liang Yin, $\mathrm{PhD}$, research interests: synthetic biology, Email: yin103@163.com; Lijuan Wang, Master, research interests: active components analysis of microalgae, Email: wljhexi@163.com.

*Corresponding author: Guanghong Luo, Master, Professor, research interests: agricultural engineering. Gansu Agricultural University, China, No.846 Beihuan Road, Ganzhou District, Zhangye 734000, Gansu, China. Tel: +8613993693452, Email: kyluo@hxu.edu.cn. should have the maximum specific growth rate and the highest $\beta$-carotene productivity under optimized conditions. However, the specific growth rate of $D$. salina is only $0.16-0.20 \mathrm{~d}^{-1[6]}$, resulting in low $\beta$-carotene productivity and this low growth rate makes the cultivation system vulnerable to biological contamination, consequently hinders large-scale cultivation.

Several attempts have been carried out to isolate mutants with straits by using chemical mutagens, ultraviolet (UV) or $\gamma$ and $\mathrm{X}$-rays exposure. For example, using UV irradiation, mutants of $D$. bardawil with higher potential for $\beta$-carotene synthesis under relatively low light intensities was obtained ${ }^{[7-9]}$. More recently, a novel mutant of $D$. salina named Zea1 was isolated by ethyl methyl sulfonate mutagenesis treatment ${ }^{[10]}$ and the zeaxanthin content of zeal improved 30-fold than the wild type. Compared with other physical methods, such as X-rays and $\gamma$-rays whose linear energy transfer values (LET; the energy transferred per unit length, $\mathrm{keV} / \mu \mathrm{m}$ ) were 0.2 and $2.0 \mathrm{keV} / \mu \mathrm{m}$, respectively ${ }^{[11]}$, the LET value of the heavy-ion beam used in this study was extremely high, reaching $31 \mathrm{keV} / \mu \mathrm{m}$. Besides, the heavy-ion beam is well known due to its higher relative biological effectiveness (RBE) compared with low-LET $\gamma$-rays and X-rays. Moreover, the heavy-ion beam can also be controlled to deposit high energy at precise positions, in contrast to low-LET irradiation that may cause large deletions, translocations or rearrangements in the genome of a given $\operatorname{organism}^{[12,13]}$. Previous studies found that heavy ions irradiation increased the biomass of Nannochloropsis by $19 \%$ and the lipid productivity of Desmodesmus sp. by $20.6 \%^{[11,13]}$, and the maximum quantum efficiency $\left(F_{v} / F_{m}\right)$ of Desmodesmus sp. and Nannochloropsis after heavy ions irradiation was also higher than the wild strains ${ }^{[11,13]}$. The microalgae Desmodesmus sp. can 
utilize nutrients such as nitrogen or phosphorous in wastewater and then produce high levels of total fatty acids, which would be a superior potential for high-quality biofuel production ${ }^{[3,14]}$. Therefore, the heavy ions irradiation may simultaneously increase biomass and by-products productivity in microalgae with improved photosynthesis activity. To the best of our knowledge, little has been reported using heavy ions irradiation on $D$. salina for efficient $\beta$-carotene production.

This work reported here is a first attempt to increase $\beta$-carotene productivity with the use of heavy-ion induced mutant produced from D. salina. Heavy-ion irradiation mutagenesis was applied in the wild type $D$. salina to improve its ability for $\beta$-carotene production. After mutation screening, one mutant (DS240G-1) was obtained, and then its biomass and $\beta$-carotene accumulation were investigated. Moreover, the underlying mechanism causing high biomass and $\beta$-carotene production were also analyzed in the DS240G-1 mutant. The chlorophyll fluorescence parameters (including the maximum quantum yield of Photosystem II $\left(F_{v} / F_{m}\right)$, the actual quantum yield of PSII (ФPSII), and non-photochemical quenching of PSII (NPQ), and pigments contents $(\beta$-carotene and chlorophyll-a content) of $D$. salina mutant were examined. Studying the effects of heavy ions exposure on the photosynthesis of microalgae will provide a better understanding of the biological effects of high-LET radiation on microalgae for $\beta$-carotene production.

\section{Materials and methods}

\subsection{Microalgae medium and cultivation}

The microalgae D. salina CCAP $19 / 18$ was purchased from Culture Collection of Algae and Protozoa (Windermere, United Kingdom). The strain was maintained in the medium of optimized Artificial Sea Water (ASW), composing of $1.5 \mathrm{~mol} / \mathrm{L}$ $\mathrm{NaCl}, 5 \mathrm{mmol} / \mathrm{L} \mathrm{KNO}, 0.45 \mathrm{mmol} / \mathrm{L} \mathrm{MgCl}_{2} \cdot 6 \mathrm{H}_{2} \mathrm{O}, 0.05 \mathrm{mmol} / \mathrm{L}$ $\mathrm{MgSO}_{4} \cdot 7 \mathrm{H}_{2} \mathrm{O}, \quad 0.3 \mathrm{mmol} / \mathrm{L} \mathrm{CaCl}_{2} \cdot 2 \mathrm{H}_{2} \mathrm{O}, 0.13 \mathrm{mmol} / \mathrm{L} \mathrm{K}_{2} \mathrm{HPO}_{4}$, $0.02 \mathrm{mmol} / \mathrm{L} \mathrm{FeCl}{ }_{3}, 0.02 \mathrm{mmol} / \mathrm{L}$ EDTA, $1 \mathrm{~mL}$ of trace elements stock per liter with $50 \mathrm{mmol} / \mathrm{L} \mathrm{H}_{3} \mathrm{BO}_{3}, 10 \mathrm{mmol} / \mathrm{L} \mathrm{MnCl}{ }_{2} \cdot 4 \mathrm{H}_{2} \mathrm{O}$, $0.8 \mathrm{mmol} / \mathrm{L} \mathrm{ZnSO} \mathrm{Zn}_{4} \cdot 7 \mathrm{H}_{2} \mathrm{O}, 0.8 \mathrm{mmol} / \mathrm{L} \mathrm{CuSO}_{4} \cdot 5 \mathrm{H}_{2} \mathrm{O}, 2 \mathrm{mmol} / \mathrm{L}$ $\mathrm{NaMoO}_{4} \cdot 2 \mathrm{H}_{2} \mathrm{O}, 1.5 \mathrm{mmol} / \mathrm{L} \mathrm{NaVO}_{3}, 0.2 \mathrm{mmol} / \mathrm{L} \mathrm{CoCl}{ }_{2} \cdot 6 \mathrm{H}_{2} \mathrm{O}$ and the $\mathrm{pH}$ was adjusted to $7.5 \mathrm{by}$ addition of Tris-buffer $(40 \mathrm{mmol} / \mathrm{L})$. D. salina seed culture was performed in $500 \mathrm{~mL}$ conical flasks at $50 \mu \mathrm{mol} /\left(\mathrm{m}^{2} \cdot \mathrm{s}\right)$ light intensity.

\subsection{Carbon-ion irradiation}

The carbon-ion irradiation was performed according to Wang et al. ${ }^{[15]}$ After adjustment of the cell concentration to $1 \times 10^{6}$ cells $/ \mathrm{mL}$, the cell suspension was exposed to carbon-ion $\left({ }^{12} \mathrm{C}^{6+}\right)$ irradiation at doses ranging from 0 to $320 \mathrm{~Gy}(0,30,60,90,120$, 180,240 and $320 \mathrm{~Gy}$ ), and there were at least three microalgae samples for each dose treatment. Irradiation was conducted at the Heavy-Ion Research Facility in Lanzhou (HIRFL), Institute of Modern Physics, Chinese Academy of Sciences. The initial energy was $80 \mathrm{MeV} / \mathrm{u}$, and the average LET was $33 \mathrm{keV} / \mu \mathrm{m}^{[15]}$. After irradiation, the microalgal cells were diluted to an appropriate concentration, spread onto ASW-agar plates in triplicate, and cultured at $(25 \pm 1)^{\circ} \mathrm{C}$ under illumination $\left(100-120 \mu \mathrm{mol}\right.$ photons $\left./ \mathrm{m}^{2} \cdot \mathrm{s}\right)$ to form colonies for mutants isolation.

\subsection{Mutant isolation and screening}

Colonies derived from the irradiated cells were selected according to their size and colors on the ASW-agar plates, and the methods were described as the Wang et al. ${ }^{[15]}$ and $\mathrm{Hu}$ et al. ${ }^{[13]}$ Firstly, individual colonies were transferred into $2 \mathrm{~mL}$ of ASW medium in the 24-well microplates and cultured for several generations to obtain purified monoclonal strains for amplification. The microalgae cells were then transferred to $10 \mathrm{~mL}$ of ASW medium in $15 \mathrm{~mL}$ glass tubes under low light $\left(50 \mu \mathrm{mol} / \mathrm{m}^{2} \cdot \mathrm{s}\right)$ at $25^{\circ} \mathrm{C}$ for $7 \mathrm{~d}$ for further screening. On Day $6, \mathrm{OD}_{680 \mathrm{~nm}}$ values of the cultures were measured with a spectrophotometer (Jasco V-530, JASCO Corporation, Japan). Afterwards, the $15 \mathrm{~mL}$ glass tubes were subjected to a high light intensity of $1000 \mu \mathrm{mol}$ photons $/ \mathrm{m}^{2} \cdot \mathrm{s}$ for another $7 \mathrm{~d}$. The $F_{v} / F_{m}$ values of microalgal mutants were then analyzed with a Water-PAM Chlorophyll Fluorometer (Water-PAM Heinz Walz GmbH, Effeltrich, Germany). Three replicates for each mutant were analyzed. After a large-scale screening, one mutant named DS240G-1 was obtained with high growth rate and photosynthesis efficiency.

\subsection{Induction of intracellular $\boldsymbol{\beta}$-carotene accumulation}

A two-stage cultivation strategy was applied for $\beta$-carotene accumulation in algal cells. Once all cultures (DS240G-1 mutant and wild type) had reached the early stationary growth phase (after $7 \mathrm{~d}$ cultivation), microalgae cells were harvested by centrifugation, followed by washing with ASW. The harvested cells were then inoculated with the same concentration in a $500 \mathrm{~mL}$ bubble column bioreactor without nitrogen. Aeration enriched with $2 \% \mathrm{CO}_{2}$ was provided at $100 \mathrm{~mL} / \mathrm{min}$. In this second stage of cultivation, $\beta$-carotene accumulation was triggered by high light intensity of $1000 \mu \mathrm{mol}$ photons $/ \mathrm{m}^{2} \cdot \mathrm{s}^{[16]}$. After cultured for $7 \mathrm{~d}$, the dry cell weight and pigment composition were measured. Triplicate experiments under each culture condition and triplicate sets of measurements were carried out.

\subsection{Analytical methods}

2.5.1 Growth analyses

Cell density was determined spectrophotometrically using a UV/VIS spectrophotometer (Jasco V-530, JASCO Corporation, Japan) at $680 \mathrm{~nm}$. The microalgal dry weight (DW) was determined according to the method described by Cao et al. ${ }^{[1]}$ Briefly, with pre-weighed Whatman GF/C filters, $10 \mathrm{~mL}$ culture broth was filtered and washed three times with $2 \mathrm{~mL}$ of $0.5 \mathrm{~mol} / \mathrm{L}$ ammonium bicarbonate and then dried below $60^{\circ} \mathrm{C}$ for over $16 \mathrm{~h}$ until the weight was constant. The DW of the microalgae cells was calculated according to the difference between the final and initial filter weight and volume of the filtered sample.

The microalgal growth rate $\left(\mu_{i}, \mathrm{~d}^{-1}\right)$ was calculated by Equation (1).

$$
\mu_{i}=\frac{L n D W_{i}-L n D W_{i-1}}{t_{i}-t_{i-1}}
$$

The biomass productivity $\left(P_{i}, \mathrm{~g} / \mathrm{L} \cdot \mathrm{d}\right)$ was calculated as

$$
P_{i}=\frac{D W_{i}-D W_{i-1}}{t_{i}-t_{i-1}}
$$

where, $D W_{i}$ and $D W_{i-1}$ are the biomass concentration measured at time $t_{i}$ and $t_{i-1}(\mathrm{~g} / \mathrm{L})$, respectively; $t_{i}$ and $t_{i-1}$ are Day $i$ and $i-1$ when the culture broth was sampled.

\subsubsection{Main cell composition extraction and estimation}

Approximately $5 \mathrm{mg}$ dry biomass (for biomass fatty acid analysis) was added to $98 \%: 2 \%$ (v/v) methanol: $\mathrm{H}_{2} \mathrm{SO}_{4}$ and incubated for $1 \mathrm{~h}$ at $70^{\circ} \mathrm{C}^{[18]}$. After methylation, deionized water and hexane were added to extract the fatty acid methyl esters (FAMEs). C17:0-TAG was added as the internal standard for quantification. Separation and identification of FAMEs were performed by using gas chromatography flame ionization detection (GC-FID, Agilent 6890) with a DB-23 capillary column (30 mm× $0.32 \mathrm{~mm} \times 0.25 \mu \mathrm{m})$. The injector temperature was $270^{\circ} \mathrm{C}$, with a split ratio of 50:1. The program for the column temperature began 
at $130^{\circ} \mathrm{C}$ for $1 \mathrm{~min}$, followed by an increase to $170^{\circ} \mathrm{C}$ at a rate of $10^{\circ} \mathrm{C} / \mathrm{min}$, then another increase at a rate of $2.8^{\circ} \mathrm{C} / \mathrm{min}$ to $215^{\circ} \mathrm{C}$, where it was maintained for $1 \mathrm{~min}$. The detection temperature was $300^{\circ} \mathrm{C}$. FAMEs were identified by using the retention time and mass spectral matching. The protein content was determined using Markwell method, a modified Lowry method ${ }^{[19]}$. The starch content was measured according to the method described by Zheng et al. ${ }^{[20]}$ Briefly, the algal pellet was re-suspended in $0.1 \mathrm{~mol} / \mathrm{L} \mathrm{pH}$ 4.4 acetate buffer, and autoclaved at $110^{\circ} \mathrm{C}$ for $15 \mathrm{~min}$ to solubilize the starch. Then 1.5 units of amyloglucosidase (Sigma-Aldrich, St, Louis, Mo, USA) were added and the solution was heated at $55^{\circ} \mathrm{C}$ for $1 \mathrm{~h}$. The glucose was determined by the sulfuric acid-anthrone method. The blank assay for starch determination was carried out by adding equal amounts of reagents to a microalgae-free sample. Starch content (\% DW) was calibrated by subtracting the glucose content from blank assay and multiplying 0.9 .

\subsubsection{Pigment extraction and determination}

To determine the contents of pigments including chlorophyll (Chla and Chlb) and total carotenoids, $10 \mathrm{mg}$ of dried biomass was extracted with $1 \mathrm{~mL} 90 \%(\mathrm{v} / \mathrm{v})$ acetone, vortexed for $20 \mathrm{~s}$, and then centrifuged at $10000 \mathrm{~g}$ for $2 \mathrm{~min}$. The above pigment extraction procedure was repeated several times until the algae were colorless. The absorbance at $645 \mathrm{~nm}\left(A_{645}\right), 662 \mathrm{~nm}\left(A_{662}\right)$, and $470 \mathrm{~nm}\left(A_{470}\right)$ of the extraction solution were measured respectively, using a UV/VIS spectrophotometer (Jasco V-530, JASCO Corporation, Japan). Chla (mg/L), Chlb (mg/L), and total carotenoids contents $(\mathrm{mg} / \mathrm{L})$ were calculated using the equations below:

$$
\begin{aligned}
\text { Chla } & =11.75\left(A_{662}\right)-2.35\left(A_{645}\right) \\
\text { Chlb } & =18.61\left(A_{645}\right)-3.96\left(A_{662}\right) \\
\text { Total carotenoids } & =\frac{1000 A_{470}-2.270 \text { Chla }-81.4 \text { Chlb }}{198}
\end{aligned}
$$

$$
\text { Pigment content }=\frac{\text { Pigment concentration } \times V}{M} \times 0.001 \times 100 \%
$$

\subsection{4 $\beta$-carotene accumulation analysis}

The modified spectrophotometric method was used to determine $\beta$-carotene content in biomass ${ }^{[21]} .1 \mathrm{~mL}$ of cell suspension was centrifuged at $10000 \mathrm{r} / \mathrm{min}$ for $2 \mathrm{~min}$. After centrifugation, the supernatant was discarded and $3 \mathrm{~mL}$ dodecane was added. The sample was shaken vigorously to re-suspend the algae pellets. Then, $9 \mathrm{~mL}$ of methanol was added to completely break up the cells and the tube was shaken vigorously again, then centrifuged for $2 \mathrm{~min}$ at $10000 \mathrm{r} / \mathrm{min}$. The dodecane-containing lipophilic carotenoids (upper layer) were measured with a spectrophotometer (Jasco V-530, JASCO Corporation, Japan) at $453 \mathrm{~nm}$ and $665 \mathrm{~nm}$ with dodecane as reference. The $\beta$-carotene concentration was calculated using Equation (7):

$$
C_{\beta \text {-car }}=\left(A_{453}-A_{665} / 3.91\right) \times 3.657 \times 3 X
$$

where, $\left(A_{453}-A_{665} / 3.91\right)$ is the absorbance of $\beta$-carotene corrected for chlorophyll contamination; 3.657 is the calibration factor derived from HPLC analysis of $\beta$-carotene concentration; 3 is the amount of milliliter of dodecane added for extraction; $X$ is the dilution factor to measure absorbance on the spectrophotometer.

The content of $\beta$-carotene in the algae biomass was calculated according to Equation (8):

$$
\beta \text {-carotene }=\frac{C_{\beta-c a r} \times 10}{D W}
$$

where, $C_{\beta \text {-car }}$ is $\beta$-carotene concentration, $\mathrm{mg} / \mathrm{L} ; \beta$-carotene is $\beta$-carotene content, $\%$; and $D W$ is cell dry weight, $\mathrm{mg} / \mathrm{L}$.

\subsubsection{Chlorophyll fluorescence measurement}

PSII activity of algal cells was measured by a chlorophyll fluorometer (Water-PAM Heinz Walz GmbH, Effeltrich, Germany). Firstly, the dark adaption of $10 \mathrm{~min}$ was performed before applying a saturating pulse $\left(0.6 \mathrm{~s}, 1400 \mu \mathrm{mol} / \mathrm{m}^{2} \cdot \mathrm{s}\right)$ to measure the maximal PSII quantum yield $\left(F_{v} / F_{m}\right)$, quantum yield of PSII (ФPSII), nonphotochemical quenching (NPQ) and relative photosynthetic electron transport rate (rETR). $F_{v} / F_{m}$, ФPSII, NPQ and rETR were determined according to the methods described by Yao et al. ${ }^{[22]}$ and Wang et al. ${ }^{[15]}$

\subsubsection{ROS Measurement}

Confocal microscopy images of cellular ROS were captured following the method described by Zheng et al. ${ }^{[20]}$ Briefly, $D$. salina cells ( $\sim 2$ million) were collected by centrifugation, washed once, and resuspended in $1 \times$ PBS buffer containing $5.0 \mu \mathrm{mol} / \mathrm{L}$ of the oxidant-sensing fluorescent probe $\mathrm{H}_{2}$ DCFDA (Thermo Fisher Scientific). After incubation at room temperature in the dark for $30 \mathrm{~min}$, the samples were washed three times with $1 \times$ PBS buffer. The DCF fluorescence of 2 million cells was also collected with a fluorescence spectrophotometer (F-4500, Hitachi, Japan) using excitation at $504 \mathrm{~nm}$ and emission at $524 \mathrm{~nm}$.

\subsection{Statistical analyses}

The One-way ANOVA analyses ${ }^{[17]}$ were performed in Excel (version 2013, Microsoft) to make the significance analysis for the growth (dry weight), fatty acid content, protein content, starch content and $\beta$-carotene content of the microalgae WT and DS240G-1.

\section{Results and discussion}

\subsection{Heavy-ion irradiation mutagenesis}

The wild $D$. salina strain was treated with a wide dose range (0-320 Gy) of heavy-ion irradiation. As shown in Figure 1, the relationship between the survival rate of $D$. salina and the irradiation dose was fitted to a logistic curve equation, showing that the survival rate of D. salina decreased by approximately $69 \%$ to $27 \%$ with the increase of radiation dose. According to the previous report in mutagenesis breeding, high mutation frequency was usually found at low survival rates ${ }^{[15]}$. The results here indicated that low doses of irradiation (0-60 Gy) gave a high survival rate but a low probability of hyper-producing mutants. By contrast, high dose of irradiation (90-320 Gy) caused irreversible damage to cells and hindered growth. Hence, $D$. salina irradiated under 90, 120, 180, 240 and $320 \mathrm{~Gy}$, respectively, were selected for further mutation screen in this study.

After carbon-ions irradiation treatment, microalgae colonies which appeared on ASW-agar plates were selected as putative mutants. The initial putative mutants screening through light microscopy revealed that the morphological characteristics (e.g. colony size, cell appearance and shape, etc.) of the assumed mutants were different from the wild-type algae cells. According to the previous study, a screening strategy of microalgae mutants with high yields of biomass and lipid-based on the determination of $F_{v} / F_{m}$ value was developed ${ }^{[13]}$. Hence, the quantum efficiency of PSII $\left(F_{v} / F_{m}\right)$ was select as an indicator to further characterization of the putative mutants by means of a chlorophyll fluorescence technology ${ }^{[13]}$. All algae mutants were subjected to the $F_{v} / F_{m}$ value analysis under different illuminations by Water-PAM firstly. Hereafter, the algal growth and $\beta$-carotene yields of the mutants with high $F_{v} / F_{m}$ values were quantified in 24-well microplates. From this method, the mutants which have $F_{v} / F_{m}$ and $\beta$-carotene 
content at least $10 \%$ higher than wild type were chosen for further study, while the other mutants were throw away ${ }^{[13]}$.

After the large-scale screening, one mutant (DS240G-1) obtained from 240 Gy of heavy-ion irradiation was selected with a growth rate $50 \%$ higher than the wild strain. The photosynthetic efficiency and $\beta$-carotene contents of DS240G-1 were also $10 \%$ higher than those of wild type. Similar results had also been reported in heavy mutagenesis which got the desired mutant from the high cell mortality condition ${ }^{[11]}$.

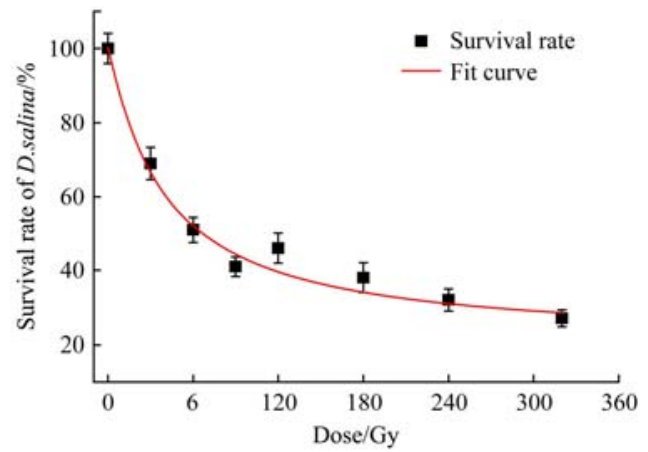

Note: Data are means of three repeated experiments and error bars indicate standard deviations.

Figure 1 Survival rate of Dunaliella salina CCAP 19/18 treated with different doses of carbon-ions irradiation

\subsection{Growth and pigment characterizations of the DS240G-1 mutant}

To further characterize the growth and pigment accumulation of DS240G-1, the growth curve, photosynthetic pigments content, and photosynthesis characteristics for the wild type and DS240G-1 were investigated in this study. Biomass production is one of the most important factors for microalgal commercialization ${ }^{[23]}$. As shown in Figure $2 \mathrm{a}$ and Table 1, the highest biomass dry weight

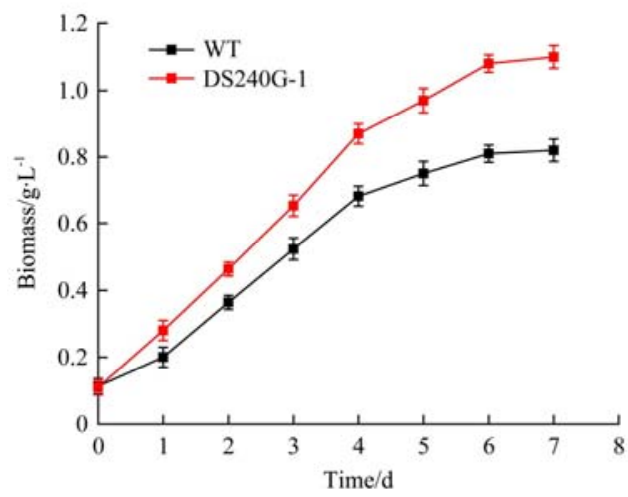

a. Growth curve

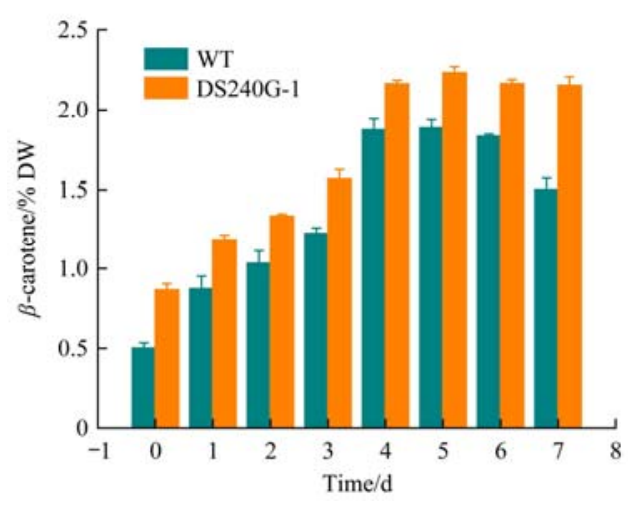

c. $\beta$-carotene content
$(1.10 \mathrm{~g} / \mathrm{L})$ and biomass productivity $(0.14 \mathrm{~g} / \mathrm{L} \cdot \mathrm{d})$ of DS240G-1 were $34 \%$ and $40 \%$ higher than those of the wild type. The maximum specific growth rate of DS240G-1 was $0.90 \mathrm{~d}^{-1}$, which was $55 \%$ higher than that of the wild type. These results indicated that DS240G-1 could be considered as a promising candidate for future commercialization.

Photosynthetic pigments are the major compositions of the light-harvesting complex which capture light energy in microalgae photosynthesis $^{[23]}$. It was shown that the chlorophyll-a content of both wild type and DS240G-1 increased after inoculation, and reached their highest level on day 3, and then decreased in the following cultivation days (Figure $2 \mathrm{~b}$ ). The chlorophyll-a content of DS240G-1 was $14 \%$ higher than that of the wild type on day 3 (Figure 2b). A similar tendency was observed in the $\beta$-carotene content of these two strains (Figure 2c). The $\beta$-carotene content of DS240G-1 was $19 \%$ higher than wild strain on day 5 . A possible explanation for higher photosynthetic pigment content is that enhanced pigment content might improve the light energy absorption ability, and then lead to the enhancement of the photosynthesis efficiency of microalgae, thus result in the biomass increasing in the DS240G-1 strain. Similar results that the enhancement of photosynthetic pigments content could increase the biomass had been reported in other microalgae strains ${ }^{[24-26]}$.

Table 1 Growth analyses of $D$. salina wild type (WT) and DS240G-1 mutant for $7 \mathrm{~d}$ cultivation

\begin{tabular}{lcc}
\hline & WT & DS240G-1 \\
\hline Dry weight $/ \mathrm{g} \cdot \mathrm{L}^{-1}$ & $0.82^{* *}( \pm 0.03)$ & $1.10^{* *}( \pm 0.02)$ \\
Productivity $/ \mathrm{g} \cdot \mathrm{L}^{-1} \cdot \mathrm{d}^{-1}$ & $0.10^{*}( \pm 0.00)$ & $0.14^{*}( \pm 0.00)$ \\
Specific growth rate/d & $0.60^{* *}( \pm 0.03)$ & $0.93^{* *}( \pm 0.02)$ \\
\hline
\end{tabular}

Note: Values are means $( \pm \mathrm{SD})$ of $n=3$ cultivations per treatment, * represent the significant effect $(p<0.05)$ and $* *$ represent the very significant effect $(p<0.01)$

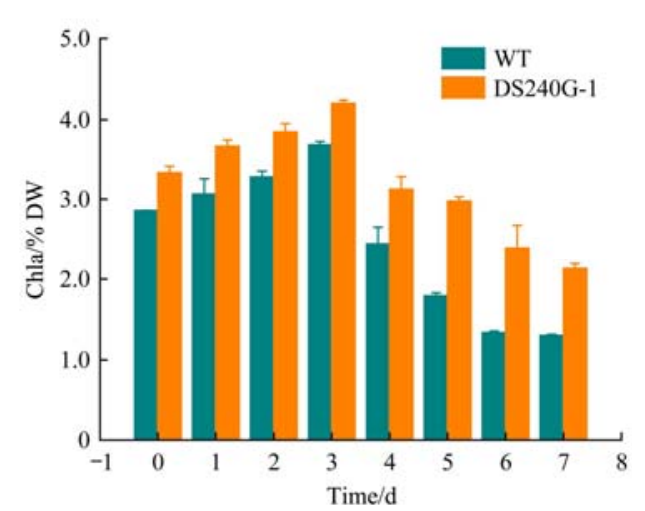

b. chlorophyll a (Chla)

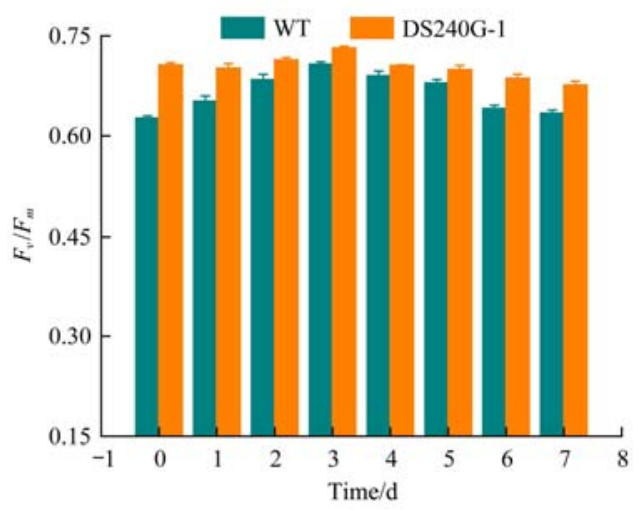

d. $F_{v} / F_{m}$

Note: Data are means of three replicates and error bars indicate standard deviations.

Figure 2 Growth curve, chlorophyll a (Chla), $\beta$-carotene content and $F_{v} / F_{m}$ of $D$. salina wild type (WT) and DS240G-1 
To further study the reason for high biomass production of DS240G-1, photosynthetic parameter $F_{v} / F_{m}$ was measured in both DS240G-1 and wild-type strains (Figure 2d). The energy absorbed by light from the photosynthetic pigment of microalgae is converted into photosynthesis during the quantum conversion process of photosynthesis, chlorophyll fluorescence and heat dissipation $^{[27]} . \quad F_{v} / F_{m}$, reflecting the potential maximum quantum efficiency, can directly reflect the photosynthesis activity of PSII ${ }^{[28,29]}$. As shown in Figure $2 d$, the average values of $F_{v} / F_{m}$ in DS240G-1 were $5 \%$ higher than that of the wild type during the entire culture process, which further verified the higher biomass was because of higher photosynthesis activity in DS240G-1.

\section{3 $\beta$-carotene accumulation of the mutant DS240G-1 with} high light

To further investigate the $\beta$-carotene accumulation of DS240G-1, a two-stage cultivation model was applied. Owing to separate growth and $\beta$-carotene production phases, the two-stage cultivation has been proved to improve overall $\beta$-carotene productivity in microalga $e^{[9]}$. In this study, after $7 \mathrm{~d}$ cultivation in low light intensity and nitrogen-repletion conditions, microalgae culture was harvested and inoculated into the second-stage culture broth under an irradiance of $1000 \mu \mathrm{mol}$ photon $/ \mathrm{m}^{2} \cdot \mathrm{s}$ with the same cell biomass $(0.5 \mathrm{~g} / \mathrm{L})$. As shown in Figure 3a, there was no significant difference $(p>0.05)$ in biomass accumulation between the DS240G-1 and wild type during the cultivation period, and the biomass of DS240G-1 (0.78 g/L) was similar with the wild type $(0.76 \mathrm{~g} / \mathrm{L})$ on Day 5 . Furthermore, the maximal $\beta$-carotene content $(8.75 \%)$ was obtained in DS240G-1 on Day 5 at the second-stage culture, which was $21 \%$ higher than that of the wild type $(7.23 \%)$ (Figure $3 b)$. Therefore, due to the significant increase of $\beta$-carotene content, the $\beta$-carotene yield of DS240G- 1 $(66.5 \mathrm{mg} / \mathrm{L})$ was $18 \%$ higher compared to the wild type $(56.4 \mathrm{mg} / \mathrm{L})$ on Day 5 at the second-stage culture (Figure $3 \mathrm{c}$ ).

To further investigate the reason for the high biomass productivity of DS240G-1 during $\beta$-carotene accumulation, the photosynthetic parameters including ФPSII, NPQ and rETR were determined. As shown in Figure 3d, on day 5 with the highest $\beta$-carotene production, the ФPSII values of DS240G-1 and the wild type decreased with increasing photosynthetically active radiation (PAR, 29-1416 $\mu \mathrm{mol}$ photons $/ \mathrm{m}^{2} \cdot \mathrm{s}$ ). The ФPSII values of DS240G-1 were higher than that of the wild strain over the entire investigated light step ranges $(p<0.05)$. The above results showed that the photosynthetic efficiency of DS240G-1 was higher than that of the wild type during the $\beta$-carotene accumulation phase, which might result in its higher biomass productivity.

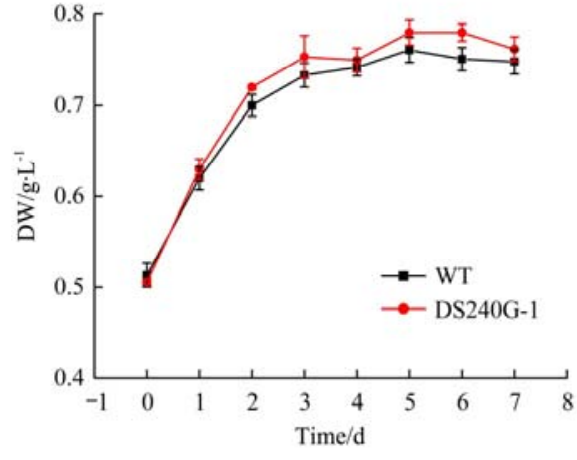

a. Growth curve

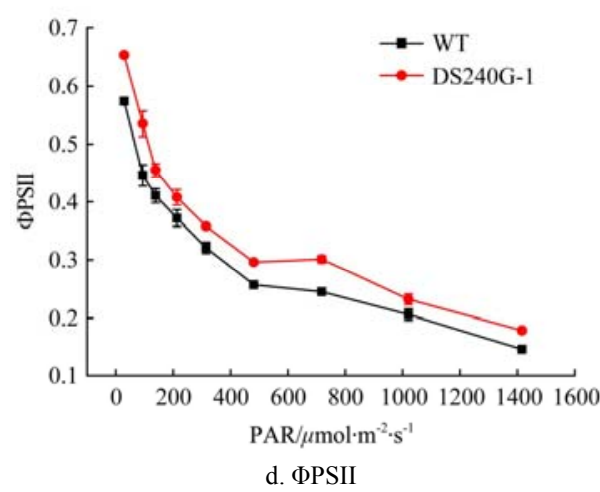

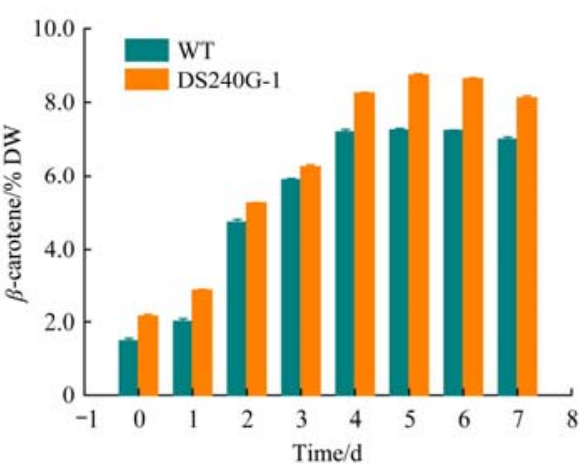

b. $\beta$-carotene content

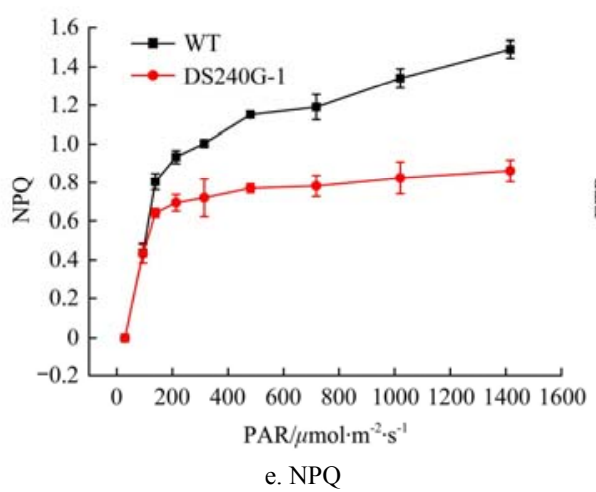

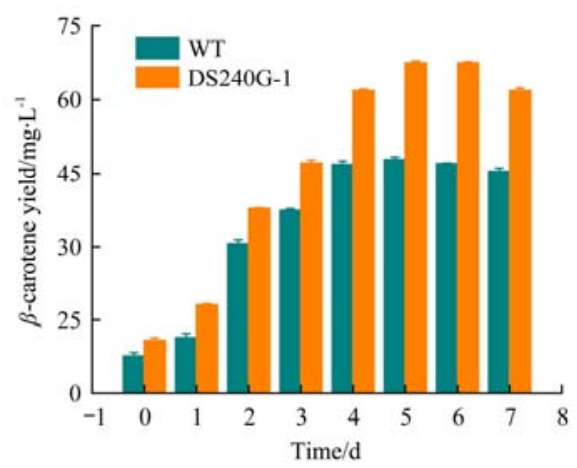

c. $\beta$-carotene productivity

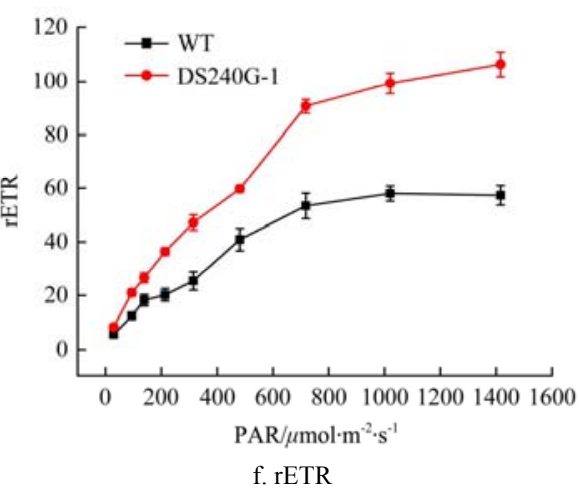

Note: Data are means of three repeated experiments and error bars indicate standard deviations

Figure 3 Cell biomass, $\beta$-carotene content and photosynthetic characteristics during $\beta$-carotene accumulation period of $D$. salina wild type (WT) and DS240G-1 mutant. Growth curve, $\beta$-carotene content and $\beta$-carotene productivity, ФPSII, NPQ, rETR.

NPQ reflects a protection process that thermally dissipates excess light energy that is not used for photosynthesis ${ }^{[15,30]}$. As shown in Figure 3e, the NPQ values of DS240G-1 and wild-type strain increased with increasing PAR. The NPQ values of DS240G-1 were lower than that of the wild-type strain over the light steps ranging from 139 to $1416 \mu \mathrm{mol}$ photons $/ \mathrm{m}^{2} \cdot \mathrm{s}(p<0.05)$, and suggesting that DS240G-1 exhibited less thermal dissipation than the wild type strain at high levels of light steps. Also, the rETR is an approximation of the rate of electrons pumped through the photosynthetic chain ${ }^{[15,28]}$. As shown in Figure 3f, the rETR values of DS240G-1 and wild type strain increased in response to increasing PAR and reached a maximum at $1416 \mu \mathrm{mol}$ photons $/\left(\mathrm{m}^{2} \cdot \mathrm{s}\right)$ and $1020 \mu \mathrm{mol}$ photons $/\left(\mathrm{m}^{2} \cdot \mathrm{s}\right)$, respectively. The rETR values of mutant DS240G-1were higher than that of the wild type strain over the light steps range $29-1416 \mu \mathrm{mol}$ photons $/ \mathrm{m}^{2} \cdot \mathrm{s}$ $(p<0.05)$, indicating that the mutant strain DS240G-1 showed higher photosynthetic electron transport rates than the wild type strain. In summary, high actual photosynthetic efficiency of PSII, low thermal dissipation ability, and high electron transport rate at all light steps $\left(29-1416 \mu \mathrm{mol}\right.$ photons $\left./ \mathrm{m}^{2} \cdot \mathrm{s}\right)$ were observed for 
mutant DS240G-1. Besides, mutant DS240G-1 showed stronger resistance to high PAR than the wild-type strain.

The above results showed that the photosynthetic efficiency of DS240G-1 was higher than that of the wild-type strain during the $\beta$-carotene accumulation phase. $\beta$-carotene yield is one of the key factors in microalgal $\beta$-carotene commercialization. The increase in $\beta$-carotene yield depends on the improvements in both biomass and $\beta$-carotene content. Similar results have been reported that higher biomass strains of Nannochloropsis and Desmodesmus sp. can be obtained through improving photosynthesis efficiency by heavy ions irradiation ${ }^{[11,13]}$. Taken together, it was suggested that increased photosynthesis efficiency of the DS240G-1 mutant resulted in its higher $\beta$-carotene yield than that of the wild type.

\subsection{Changes in ROS content and cell biomass composition of the mutant with high light}

When wild type and DS240G-1 were cultivated under high light (HL) conditions, the ROS content of DS240G-1 increased dramatically in the first-day cultivation. From day 2 to day 7, the ROS content decreased with the increasing $\beta$-carotene content. The ROS content of DS240G-1 was higher than (20\%-94\%) that of the wild strain over the $\beta$-carotene accumulation stage. Under stress conditions including high light irradiation, and nitrogen deficiency, ROS are generated in D. salina ${ }^{[20]}$, which can lead to cellular damage. In photosynthetic organisms, in order to avoid or

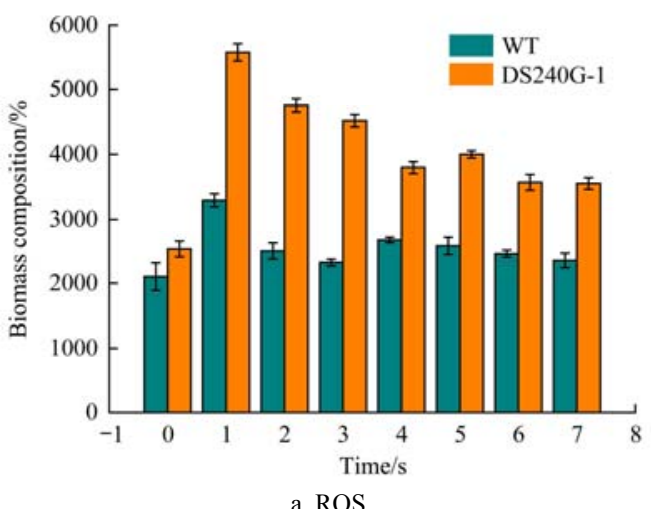

relieve the damage caused by ROS, photosynthetic organisms have evolved different types of mechanisms to scavenge $\operatorname{ROS}^{[20]}$. In microalgae $D$. salina, $\beta$-carotene, as an integral component of microalgae photosynthetic apparatus pigment, can protect cells against the ROS formed from photo-oxidation ${ }^{[20,31]}$. The result of this study revealed that high content of ROS might induce massive $\beta$-carotene accumulation in mutant DS240G-1.

Furthermore, the total fatty acid, protein and starch contents were determined in wild type and DS240G-1 under high light conditions (Table 2 and Figure 4). The protein content in wild-type strain was $21 \%$ higher than that in mutant DS240G-1, but the starch content in wild-type strain was $22 \%$ lower than that in mutant DS240G-1. Also, the total fatty acid (TFA) content in mutant DS240G-1 (approximately 18\% DW) was 79\% higher than in wild type. Owing to its higher $\beta$-carotene productivity and higher TFA content, DS240G-1 could be considered as a promising candidate for microalgae $\beta$-carotene and biodiesel production.

Table 2 Major biomass compositions of WT and DS240G-1

\begin{tabular}{cccc}
\hline Strains & Protein/\%DW & Starch/\%DW & TFA/\%DW \\
\hline WT & $48.51^{*}( \pm 4.10)$ & $13.40( \pm 1.61)$ & $9.85^{* *}( \pm 0.83)$ \\
DS240G-1 & $40.13^{*}( \pm 1.62)$ & $16.30( \pm 2.15)$ & $18.43^{* *}( \pm 0.61)$ \\
\hline
\end{tabular}

Note: Values are means $( \pm \mathrm{SD})$ of $n=3$ cultivations per treatment, $*$ represent the significant effect $(p<0.05)$ and $* *$ represent the very significant effect $(p<0.01)$.

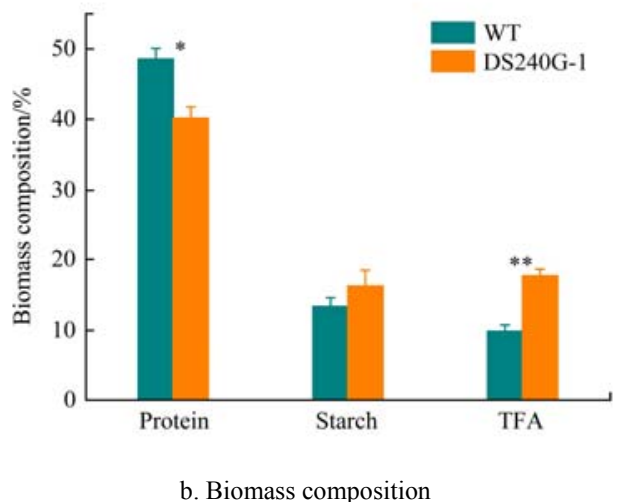

Note: Data are means of three repeated experiments and error bars indicate standard deviations, *represent the significant effect $(p<0.05)$ and ** represent the very significant effect $(p<0.01)$

Figure 4 ROS and biomass composition characteristics during $\beta$-carotene accumulation period of $D$. salina wild type (WT) and DS240G-1 mutant

\section{Conclusions}

This work presented the first attempt to use carbon ions to induce mutagenesis of microalgae for enhanced $\beta$-carotene productivity. In this study, the mutant (DS240G-1) with high $\beta$-carotene productivity $(67.6 \mathrm{mg} / \mathrm{L})$ was obtained from $D$. salina. Compared to the wild strain, its $\beta$-carotene content and TFA content increased by $21 \%$ and $79 \%$, respectively, likely owing to the improved quantum efficiency of photosynthesis and ROS content under HL stress conditions, and indicated that the mutant strain could be considered as a valuable candidate for microalgal $\beta$-carotene production. More work remains to be done to clarify the molecular mechanism of mutant (DS240G-1) strain after carbon ions irradiation.

\section{Acknowledgements}

This work was supported by the National Nature Science Foundation of China (Grant No. 11665011), and Gansu Provincial Science and Technology Program (Grant No. 18JR2JG001).

\section{[References]}

[1] Li G, Ji F, Bai X, Zhou Y G, Dong R J, Huang Z G. Comparative study on thermal cracking characteristics and bio-oil production from different microalgae using Py-GC/MS. Int J Agric \& Biol Eng, 2019; 12(1): 208-213.

[2] Li G, Bai X, Huo S H, Huang Z G. Fast pyrolysis of LERDADEs for renewable biofuels. IET Renewable Power Generation, 2020; 14(6): 959-967.

[3] Li G, Zhang J, Li H, Hu R C, Yao X L, Liu Y, et al. Towards high-quality biodiesel production from microalgae using original and anaerobically-digested livestock wastewater. Chemosphere, 2020; 128578.

[4] Li G, Lu Z T, Zhang J, Li H, Zhou Y G, Zayan A M I, et al. Life cycle assessment of biofuel production from microalgae cultivated in anaerobic digested wastewater. Int J Agric \& Biol Eng, 2020; 13(1): 241-246.

[5] Mojaat M, Pruvost J, Foucault A, Legrand J. Effect of organic carbon sources and $\mathrm{Fe}^{2+}$ ions on growth and beta-carotene accumulation by Dunaliella salina. Biochemical Engineering Journal, 2008; 39: 177-184.

[6] Tafreshi A H, Shariati M. Dunaliella biotechnology: Methods and applications. Journal of Applied Microbiology, 2009; 107: 14-35.

[7] Shaish A, Benamotz A, Avron M. Production and selection of high beta-carotene mutants of Dunaliella-bardawil (chlorophyta). Journal of Phycology, 1991; 27: 652-656. 
[8] Mogedas B, Casal C, Forjan E, Vilchez C. Beta-Carotene production enhancement by UV-A radiation in Dunaliella bardawil cultivated in laboratory reactors. Journal of Bioscience And Bioengineering, 2009; 108: $47-51$.

[9] Gong M Y, Bassi A. Carotenoids from microalgae: A review of recent developments. Biotechnology Advances, 2016; 34: 1396-1412.

[10] Jin E S, Feth B, Melis A. A mutant of the green alga Dunaliella salina constitutively accumulates zeaxanthin under all growth conditions. Biotechnology and Bioengineering, 2003; 81: 115-124.

[11] Ma Y, Wang Z, Zhu M, Yu C, Cao Y, Zhang D, et al. Increased lipid productivity and TAG content in Nannochloropsis by heavy-ion irradiation mutagenesis. Bioresource Technology, 2013; 136: 360-367.

[12] Kamalanathan M, Ly H T D, Chaisutyakorna P, Gleadow R, Beardall J. Photosynthetic physiology of Scenedesmus sp. (Chlorophyceae) under photoautotrophic and molasses-based heterotrophic and mixotrophic conditions. Phycologia, 2017; 56: 666-674.

[13] Hu G, Fan Y, Zhang L, Yuan C, Wang J, Li W, et al. Enhanced lipid productivity and photosynthesis efficiency in a Desmodesmus sp. mutant induced by heavy carbon ions. Plos One, 2013; 8: 1-11.

[14] Li G, Bai X, Li H, Lu Z T, Zhou Y G, Wang Y K, et al. Nutrients removal and biomass production from anaerobic digested effluent by microalgae: A review. Int J Agric \& Biol Eng, 2019; 12(5): 8-13.

[15] Wang J, Li X, Lu D, Du Y, Ma L, Li W, et al. Photosynthetic effect in Selenastrum capricornutum progeny after carbon-ion irradiation. Plos One, 2016; 11: 1-11.

[16] Lamers P P, Janssen M, de Vos R C H, Bino R J, Wijffels R H. Carotenoid and fatty acid metabolism in nitrogen-starved Dunaliella salina, a unicellular green microalga. Journal of Biotechnology, 2012; 162: $21-27$.

[17] Cao X P, Xi Y M, Liu J, Chu Y D, Wu P C, Yang M, et al. New insights into the $\mathrm{CO}_{2}$-steady and $\mathrm{pH}$-steady cultivations of two microalgae based on continuous online parameter monitoring. Algal Research-Biomass Biofuels and Bioproducts, 2019; 38: 1-6.

[18] Liu J, Liu Y, Wang H, Xue S. Direct transesterification of fresh microalgal cells. Bioresource Technology, 2015; 176: 284-287.

[19] Sui Y X, Muys M, Vermeir P, D'Adamo S, Vlaeminck S E. Light regime and growth phase affect the microalgal production of protein quantity and quality with Dunaliella salina. Bioresource Technology, 2019; 275: 145-152.
[20] Zheng Z B, Gao S, He Y, Li Z Y, Li Y X, Cai X H, et al. The enhancement of the oxidative pentose phosphate pathway maybe involved in resolving imbalance between photosystem I and II in Dunaliella salina. Algal Research-Biomass Biofuels and Bioproducts, 2017; 26: 402-408.

[21] Zhu C B, Zhai X Q, Jia J, Wang J H, Han D S, Li Y H, et al. Seawater desalination concentrate for cultivation of Dunaliella salina with floating photobioreactor to produce beta-carotene. Algal Research-Biomass Biofuels and Bioproducts, 2018; 35: 319-324.

[22] Yao C H, Ai J N, Cao X P, Xue S, Zhang W. Enhancing starch production of a marine green microalga Tetraselmis subcordiformis through nutrient limitation. Bioresource Technology, 2012; 118: 438-444.

[23] Williams P L, Laurens L L. Microalgae as biodiesel \& biomass feedstocks: Review \& analysis of the biochemistry, energetics \& economics. Energy \& Environmental Science, 2010; 3: 554-590.

[24] Qiang H, Zarmi Y, Richmond A. Combined effects of light intensity, light-path and culture density on output rate of Spirulina platensis (Cyanobacteria). European Journal of Phycology, 1998; 33: 165-171.

[25] Manandhar-Shrestha K, Hildebrand M. Development of flow cytometric procedures for the efficient isolation of improved lipid accumulation mutants in a Chlorella sp microalga. Journal of Applied Phycology, 2013, 25: $1643-1651$.

[26] Anandarajah K, Mahendraperumal G, Sommerfeld M, Hu Q. Characterization of microalga Nannochloropsis sp. mutants for improved production of biofuels. Applied Energy, 2012; 96: 371-377.

[27] White S, Anandraj A, Bux F. PAM fluorometry as a tool to assess microalgal nutrient stress and monitor cellular neutral lipids. Bioresource Technology, 2011; 102: 1675-1682.

[28] Havlik I, Reardon K F, Uenal M, Lindner P, Prediger A, Babitzky A, et al. Monitoring of microalgal cultivations with on-line, flow-through microscopy. Algal Research-Biomass Biofuels and Bioproducts, 2013; 2: 253-257.

[29] Obata M, Toda T, Taguchi S. Using chlorophyll fluorescence to monitor yields of microalgal production. Journal of Applied Phycology, 2009; 21: 315-319.

[30] MacIntyre H L, Kana T M, Anning T, Geider R J. Photoacclimation of photosynthesis irradiance response curves and photosynthetic pigments in microalgae and cyanobacteria. Journal of Phycology, 2002; 38: 17-38.

[31] Xu Y, Harvey P J. Carotenoid Production by Dunaliella salina under Red Light. Antioxidants, 2019; 8: 1-11. 